\title{
At Least Five Major Genes Are Involved in the Avirulence of an Eleusine Isolate of Pyricularia oryzae on Common Wheat
}

\author{
Soichiro Asuke, Shuko Nishimi, and Yukio Tosa ${ }^{\dagger}$ \\ Graduate School of Agricultural Science, Kobe University, Kobe 657-8501, Japan \\ Accepted for publication 23 September 2019.
}

\begin{abstract}
Pyricularia oryzae is composed of pathotypes that show host specificity at the plant genus level. To elucidate the genetic mechanisms of the incompatibility between the Eleusine pathotype (pathogenic on finger millet) and common wheat, an Eleusine isolate (MZ5-1-6) was crossed with a Triticum isolate $(\mathrm{Br} 48)$ pathogenic on wheat, and resulting $\mathrm{F}_{1}$ cultures were sprayed onto common wheat cultivars Hope, Norin 4 (N4), and Chinese Spring (CS). On Hope, avirulent and virulent cultures segregated in a 3:1 ratio, suggesting that two avirulence genes are involved. They were tentatively designated as $e A 1$ and $e A 2$. On N4 and $\mathrm{CS}$, the segregation ratio was not significantly deviated from the 7:1, 15:1, or 31:1 ratios, suggesting that three or more genes are involved. A comparative analysis of the segregation patterns suggested that two of these genes were $e A 1$ and $e A 2$. A
\end{abstract}

ABSTRACT

Pyricularia oryzae (Zhang et al. 2016) (syn. Magnaporthe oryzae), a causal agent of blast disease of various gramineous plants, is composed of several groups with restricted host ranges at the plant genus level, such as Oryza isolates pathogenic on Oryza spp. including rice (O. sativa), Setaria isolates pathogenic on Setaria spp. including foxtail millet (S. italica), Eleusine isolates pathogenic on Eleusine spp. including finger millet (E. coracana), Triticum isolates pathogenic on Triticum spp. including common wheat (T. aestivum), and Lolium isolates pathogenic on Lolium spp. including annual ryegrass (L. multiflorum) and perennial ryegrass (L. perenne) (Tosa et al. 2004). Kato et al. (2000) designated these genus-specific groups as pathotypes. They are now abbreviated as $\mathrm{MoO}$ (M. oryzae Oryza pathotype), MoS, MoE, MoT, and MoL, respectively (Cruz and Valent 2017). Gladieux et al. (2018) showed that $\mathrm{MoO}, \mathrm{MoS}, \mathrm{MoT}$, and MoL each corresponded to a single lineage in a dendrogram of $P$. oryzae isolates that was constructed using whole genome sequences.

MoT, the causal agent of wheat blast, is a new pathotype that evolved in the 1980s. Wheat blast was first reported in 1985 in the southern region of Brazil (Urashima et al. 1993). It then spread to neighboring countries such as Bolivia, Paraguay, and Argentina and became a major threat to wheat production in South America (Cruz and Valent 2017). Wheat blast showed a peculiar pattern of disease progress; almost no or only sparse symptoms were observed on leaves during the vegetative stage, but severe symptoms suddenly appeared on spikes at the heading stage. In 2016, a wheat blast outbreak occurred in Bangladesh and caused a serious loss of wheat

†Corresponding author: Y. Tosa; tosayuki@kobe-u.ac.jp

Funding: This work was supported by the Japan Society for the Promotion of Science (grant 17H01462).

*The $\boldsymbol{e}$-Xtra logo stands for "electronic extra" and indicates that one supplementary figure and one supplementary table are published online.

The author(s) declare no conflict of interest.

C 2020 The American Phytopathological Society complementation test indicated that the third gene (tentatively designated as eA3) was the Ao9 type of the PWT3 gene controlling the avirulence of Avena and Lolium isolates on wheat. The fourth gene (tentatively designated as $e A 4$ ) was detected by backcrossing $200 \mathrm{R} 72$, an $\mathrm{F}_{1}$ culture lacking $e A l$, $e A 2$, and $e A 3$, with $\mathrm{Br} 48$. Comparative analyses of phenotypes and the presence and/or absence of molecular markers in the $\mathrm{F}_{1}$ population revealed that some cultures were avirulent on N4/CS in spite of lacking $e A 1, e A 2$, $e A 3$, and $e A 4$, indicating the presence of the fifth gene (tentatively designated as $e A 5$ ). Taken together, we conclude that at least five avirulence genes are involved in the incompatibility between MZ5-1-6 and N4/CS.

Keywords: genetics and resistance production (Callaway 2016). Phylogenomic analyses revealed that this outbreak was caused by a wheat-infecting strain from South America (Islam et al. 2016; Malaker et al. 2016). Wheat blast is now becoming a pandemic disease and poses a serious threat to world wheat production. One question is how MoT, the causal agent of this devastating disease, evolved in Brazil or how its ancestral strain overcame the resistance of wheat.

Genetic mechanisms of the incompatibility between various pathotypes and wheat have been elucidated by Tosa and coworkers. Takabayashi et al. (2002) showed that two major genes were involved in the avirulence of an Avena isolate (Br58, isolated from oat) on wheat and designated them as PWT3 and PWT4. They also identified a resistance gene corresponding to PWT4 in wheat and designated it as Rmgl (syn. Rwt4). Based on these results, they suggested that the pathotype-genus specificity is conditioned by gene-for-gene interactions. Similarly, Vy et al. (2014) showed that two major genes were involved in the avirulence of a Lolium isolate (TP2, isolated from perennial ryegrass) on wheat and designated them tentatively as $A 1$ and $A 2$. Al played a major role in the avirulence, whereas $A 2$ played a minor role and was ineffective at high temperature or at the heading stage. Vy et al. (2014) also identified a resistance gene corresponding to $A l$ and designated it as Rmg6. Subsequently, Inoue et al. (2017) found that $A l$ was identical to $P W T 3$ and listed $R w t 3$ as a synonym of Rmg6. Interestingly, when $P W T 3$ of the Lolium isolate was disrupted, the disruptant gained pathogenicity on wheat spikes (Inoue et al. 2017). Based on this result, Inoue et al. (2017) suggested that MoT in Brazil may have evolved from Lolium isolates or their relatives through loss of function of PWT3. In addition, when PWT3 and PWT4 of the Avena isolate was disrupted, the double disruptant gained pathogenicity on wheat spikes (Inoue et al. 2017), suggesting that loss of function of the two avirulence genes may lead to host jump of Avena isolates to wheat. These results may raise a serious concern about the possibility that $\mathrm{MoE}$ and $\mathrm{MoO}$, which are widely distributed around the world, may also jump to wheat.

$\mathrm{MoE}$ is an "old" pathotype that is considered to have been established long ago (Kato et al. 2000). MoE isolates maintain high fertility with any other $P$. oryzae isolates, which contributed to the 
discovery of the sexual stage [a teleomorph first designated as M. grisea (Barr 1977) and later as M. oryzae (Couch and Kohn 2002)] of P. oryzae (Kato et al. 1976; Ueyama and Tsuda 1975; Yaegashi and Nishihara 1976). It consists of closely associated two lineages (Eleusine 1 and Eleusine 2 lineages in Gladieux et al. 2018 and EC-I and EC-II in Tanaka et al. 2009), which are relatively remote from MoT and MoL in a phylogenetic tree (Gladieux et al. 2018). Here, we show that at least five major genes are involved in the incompatibility between this "remote" pathotype and common wheat.

\section{MATERIALS AND METHODS}

Fungal materials. Pyricularia strains used in this study were 34 EC-II isolates from finger millet (Eleusine spp.) collected in five countries (Japan, China, Nepal, India, and Uganda), two isolates from common wheat (T. aestivum) collected in Brazil, and an isolate from oat (A. sativa) collected in Brazil (Table 1). MZ5-1-6 (Ec-3J, an Eleusine isolate belonging to EC-II with MAT1-2) was crossed with $\mathrm{Br} 48$ (T-4B, a Triticum isolate with MAT1-1) on oatmeal agar media as described in Murakami et al. (2000). One ascospore was isolated from each ascus, resulting in a total of 80 random $F_{1}$ cultures. 200R72, an $\mathrm{F}_{1}$ culture with $M A T 1$-2, was backcrossed with $\mathrm{Br} 48$ on oatmeal agar media to produce a $\mathrm{BC}_{1} \mathrm{~F}_{1}$ population. One ascospore was isolated from each ascus, resulting in a total of 60 $\mathrm{BC}_{1} \mathrm{~F}_{1}$ cultures.

Plant materials. Three cultivars of common wheat (T. aestivum) were used for infection assays: Hope, Norin 4 (N4), and Chinese Spring (CS). Nigrate (Ngt), a highly susceptible cultivar of barley
(Hordeum vulgare), was used as a control in the infection assay and as a host plant in expression analyses.

Infection assay. Wheat and barley seeds were pregerminated on moistened filter papers for $24 \mathrm{~h}$, sown in vermiculite supplied with liquid fertilizer in a seeding case $(5.5 \times 15 \times 10 \mathrm{~cm})$, and grown at $22^{\circ} \mathrm{C}$ in a controlled-environment room with a 12 -h photoperiod of fluorescent lighting for 8 days. Primary leaves of the 9-day-old seedlings were fixed onto a hard plastic board with rubber bands just before inoculation. Inocula $\left(1 \times 10^{5}\right.$ conidia/ml $)$ were prepared as described by Tagle et al. (2015). The conidial suspension was sprayed onto primary leaves using an air compressor. The inoculated seedlings were incubated in dark and humid conditions at 22 to $25^{\circ} \mathrm{C}$ for $24 \mathrm{~h}$, then returned to dry conditions with fluorescent lighting and incubated for an additional 3 to 5 days at 22 to $25^{\circ} \mathrm{C}$. Four to six days after inoculation, symptoms (infection types) were evaluated on the basis of the size and color of lesions (Hyon et al. 2012). The size was rated on six progressive grades from 0 to 5 as follows: 0 , no visible infection; 1 , pinhead spots; 2 , small lesions $(<1.5 \mathrm{~mm}) ; 3$, scattered lesions of intermediate size ( $<3 \mathrm{~mm}$ ); 4, large typical lesion; and 5, complete blighting of leaf blades. A disease score comprised a number denoting the lesion size and a letter indicating the lesion color: $\mathrm{B}$ for brown and $\mathrm{G}$ for green.

Transformation of $\boldsymbol{P}$. oryzae. A 1,068-bp DNA fragment containing the Ao' type of $P W T 3$ (Inoue et al. 2017) was amplified from genomic DNA of MZ5-1-6 with primers PWT3-UP-F and PWT3-DOWN-R (Table 2) using KOD Plus polymerase (TOYOBO, Osaka, Japan) under the following conditions: $2 \mathrm{~min}$ at $94^{\circ} \mathrm{C}$, followed by 30 cycles of $10 \mathrm{~s}$ at $98^{\circ} \mathrm{C}, 30 \mathrm{~s}$ at $60^{\circ} \mathrm{C}$, and $1 \mathrm{~min}$ at $68^{\circ} \mathrm{C}$. The amplicon was inserted into the EcoRV site of pBluescriptIISK(+).

TABLE 1. Pyricularia oryzae isolates used in this study with genotypes at the PWT3 locus

\begin{tabular}{|c|c|c|c|c|c|c|c|}
\hline Isolate & Code & Host & Locality & Year of isolation & Collector & $P W T 3$ genotype $^{\mathrm{a}}$ & Group $^{b}$ \\
\hline G10-1 & Ec-1J & Eleusine coracana & Tochigi, Japan & 1977 & S. Itoi & Ao & EC-II \\
\hline Z2-1 & Ec-2J & E. coracana & Kagawa, Japan & 1977 & S. Itoi & $\mathrm{Ao}^{\prime}$ & EC-II \\
\hline MZ5-1-6 & Ec-3J & E. coracana & Miyazaki, Japan & 1976 & H. Kato & $\mathrm{Ao}^{\prime}$ & EC-II \\
\hline KEN15-15-1 & Ec-4J & E. coracana & Tokyo, Japan & 1976 & H. Kato & $\mathrm{Ao}^{\prime}$ & EC-II \\
\hline IN77-31-1-1 & Ec-7I & E. coracana & Bangalor, India & 1977 & H. Kato & Ao & EC-II \\
\hline IN77-39-1-2 & Ec-8I & E. coracana & Mandya, India & 1977 & H. Kato & Ao & EC-II \\
\hline NP10-17-4-1-3 & Ec-9N & E. coracana & Syabru, Nepal & 1975 & S. Sakamoto & Ao & EC-II \\
\hline NP10-28-1-1-1 & Ec-10N & E. coracana & Pokhara, Nepal & 1975 & S. Sakamoto & Ao & EC-II \\
\hline NI1006 & Ea-11J & E. africana & Tochigi, Japan & 1975 & N. Nishihara & $\mathrm{Ao}^{\prime}$ & EC-II \\
\hline NI1011 & $\mathrm{Eb}-12 \mathrm{~J}$ & E. boranensis & Tochigi, Japan & 1975 & N. Nishihara & $\mathrm{Ao}^{\prime}$ & EC-II \\
\hline IN77-36-1-1 & Ei-13I & E. indica & Chammdi Hill, India & 1977 & H. Kato & Ao & EC-II \\
\hline NI1005 & Ei-101J & E. indica & Tochigi, Japan & 1975 & N. Nishihara & $\mathrm{Ao}^{\prime}$ & EC-II \\
\hline NI915 & Ei-102J & E. indica & Chiba, Japan & 1974 & N. Nishihara & $\mathrm{Ao}^{\prime}$ & EC-II \\
\hline KEN8-2 & Ec-103J & E. coracana & Tokyo, Japan & 1975 & H. Kato & $\mathrm{Ao}^{\prime}$ & EC-II \\
\hline SO4-3-2 & Ec-104J & E. coracana & Tochigi, Japan & 1975 & H. Kato & $\mathrm{Ao}^{\prime}$ & EC-II \\
\hline CH90-1-1 & Ec-107C & E. coracana & Yunnan, China & 1988 & M. Iwano & Ao & EC-II \\
\hline CH90-2-1 & Ec-108C & E. coracana & Yunnan, China & 1988 & M. Iwano & Ao & EC-II \\
\hline IN77-13-1-1 & Ebb-110I & Bambusa sp. & Ranganathittu, India & 1977 & H. Kato & Ao & EC-II \\
\hline IN77-14-1-1 & Ebb-111I & Bambusa sp. & Ranganathittu, India & 1977 & H. Kato & Ao & EC-II \\
\hline IN77-15-1-1 & Ebb-112I & Bambusa sp. & Ranganathittu, India & 1977 & H. Kato & Ao & EC-II \\
\hline IN77-23-1-1 & Ec-113I & E. coracana & Mysore, India & 1977 & H. Kato & Ao & EC-II \\
\hline IN77-24-1-1 & Ec-114I & E. coracana & Mysore, India & 1977 & H. Kato & Ao & EC-II \\
\hline IN77-25-1-1 & Ec-115I & E. coracana & Mysore, India & 1977 & H. Kato & Ao & EC-II \\
\hline IN77-30-1-1 & Ec-116I & E. coracana & Mysore, India & 1977 & H. Kato & Ao & EC-II \\
\hline IN77-40-1-1 & Ec-118I & E. coracana & Mysore, India & 1977 & H. Kato & Ao & EC-II \\
\hline IN77-41-1-3 & Ec-119I & E. coracana & Mysore, India & 1977 & H. Kato & Ao & EC-II \\
\hline IN77-42-1-1 & Ec-120I & E. coracana & Mysore, India & 1977 & H. Kato & Ao & EC-II \\
\hline IN77-43-1-1 & Ec-121I & E. coracana & Mysore, India & 1977 & H. Kato & Ao & EC-II \\
\hline IN77-44-1-1 & Ec-122I & E. coracana & Bangalor, India & 1977 & H. Kato & Ao & EC-II \\
\hline IN77-47-1-2 & Ec-123I & E. coracana & Mysore, India & 1977 & H. Kato & Ao & EC-II \\
\hline IN77-48-1-2 & Ei-124I & E. indica & Mysore, India & 1977 & H. Kato & Ao & EC-II \\
\hline IN77-49-1-1 & Ec-125I & E. coracana & Mysore, India & 1977 & H. Kato & Ao & EC-II \\
\hline NP10-10-1-1-1 & Ec- $126 \mathrm{~N}$ & E. coracana & Bhargu, Nepal & 1975 & S. Sakamoto & Ao & EC-II \\
\hline NP10-10-1-2-1 & Ec-127N & E. coracana & Bhargu, Nepal & 1975 & S. Sakamoto & Ao & EC-II \\
\hline $\mathrm{Br} 48$ & $\mathrm{~T}-4 \mathrm{~B}$ & Triticum aestivum & Mato Grosso do Sul, Brazil & 1990 & S. Igarashi & B & - \\
\hline Br116.5 & $\mathrm{T}-7 \mathrm{~B}$ & T. aestivum & Parana, Brazil & 1992 & A. S. Urashima & Atm & - \\
\hline Br58 & A-1B & Avena sativa & Parana, Brazil & 1990 & S. Igarashi & Ao & - \\
\hline
\end{tabular}

${ }^{a}$ Defined by Inoue et al. (2017).

b Defined by Tanaka et al. (2009). 
Then the recombinant plasmid or the empty vector was introduced into $\mathrm{Br} 48$ by cotransformation with pSH75 containing the hygromycin B phosphotransferase gene as described by Tosa et al. (2005). The presence and/or absence of the transgene in transformants was checked by PCR-restriction fragment length polymorphism. The $P W T 3$ genes (the endogenous B type in $\mathrm{Br} 48$ and the Ao' type in the transgene) were amplified with PWT3-HinfI-2F and PWT3-HinfI-2R (Table 2) from genomic DNA of the transformants. Amplicons obtained were digested with HinfI whose target site was present in the B type but absent in the Ao' type.

Sequence analysis of $P W T 3$ in Eleusine isolates. The $P W T 3$ region was amplified from 34 EC-II isolates (Table 1) with PWT3-UP-F and PWT3-DOWN-R as described above. The amplicon was purified by ethanol precipitation and then sequenced with the same primers as in the amplification using the BigDye Terminator version 3.1 cycle sequencing kit and an ABI 3100 or 3130xl Genetic Analyzer (Applied Biosystems, Foster City, CA) at the Kobe University Biosignal Research Center.

Expression analysis. Expression levels of three different types of PWT3 (Inoue et al. 2017) were analyzed using quantitative realtime PCR (qRT-PCR). Susceptible barley cultivar Ngt was grown for 9 days as described in the infection assay section. Conidia of MZ5-1-6 (Eleusine isolate with the Ao' type), Br58 (Avena isolate with the Ao type), and Br116.5 (Triticum isolate with the Atm type) were sprayed onto the 9-day-old primary leaves. Thirty hours after inoculation, 10 inoculated leaves were collected, assembled, and ground in liquid nitrogen using a mortar and pestle. Total RNA was extracted with Sepasol-RNA I Super G (Nakalai Tesque, Kyoto, Japan) and treated with DNase I (amplification grade; Invitrogen). cDNA was synthesized using the PrimeScript RT reagent kit (Perfect Real Time; Takara, Shiga, Japan) and oligo-dT primers according to the manufacturer's instructions. qRT-PCR was performed using FastStart SYBR Green Master (Roche, Mannheim, Germany) and the Thermal Cycler Dice Real Time System (Takara) with PWT3-specific primers and primers for actin (MGG_03982)

TABLE 4. Combined segregation of reactions on wheat cultivars in random $F_{1}$ cultures derived from MZ5-1-6 $\times$ Br48

\begin{tabular}{lcccccc}
\hline Cultivar $^{\mathrm{a}}$ & & \multicolumn{5}{c}{$\mathrm{F}_{1}$ culture $(n)^{\mathrm{b}}$} \\
\cline { 4 - 7 } $\mathrm{p}$ & $\mathrm{q}$ & $\mathrm{ApAq}$ & $\mathrm{ApVq}$ & $\mathrm{VpAq}$ & $\mathrm{VpVq}$ & Total \\
\hline N4 & $\mathrm{CS}$ & 75 & 0 & 0 & 5 & 80 \\
Hope & $\mathrm{N} 4$ & 62 & 0 & 13 & 5 & 80 \\
Hope & $\mathrm{CS}$ & 62 & 0 & 13 & 5 & 80 \\
\hline
\end{tabular}

${ }^{a} \mathrm{~N} 4=$ Norin 4 and $\mathrm{CS}=$ Chinese Spring.

b $\mathrm{A}=$ avirulent (infection type 0 to $3 \mathrm{BG}$ ) and $\mathrm{V}=$ virulent (infection type $4 \mathrm{G}$ to $5 \mathrm{G})$. For example, $A p V q$ represents cultures avirulent on $p$ and virulent on $q$.

TABLE 2. Primers used in this study

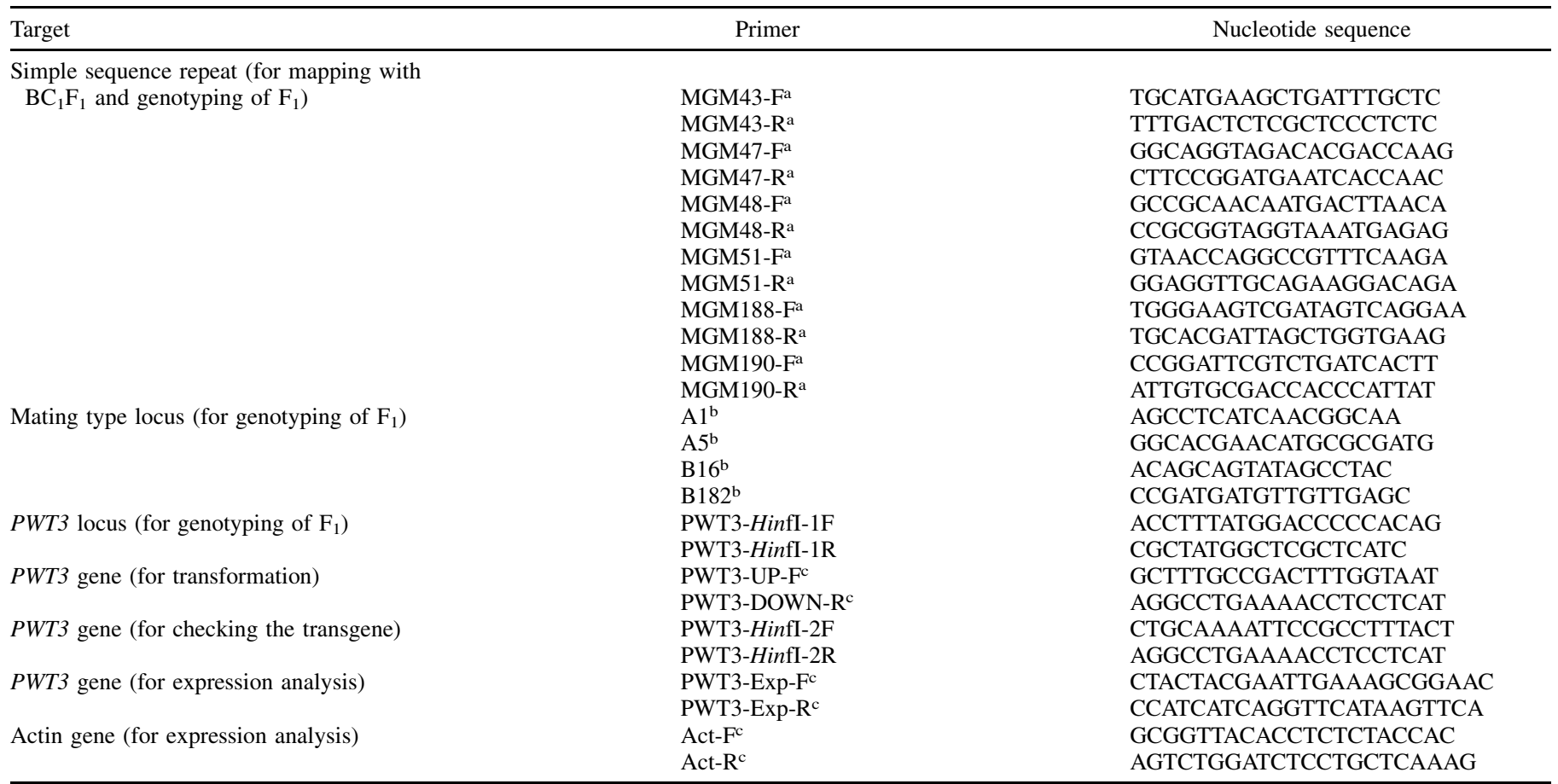

a Zheng et al. (2008). $\mathrm{F}=$ forward and $\mathrm{R}=$ reverse.

b Kanamori et al. (2007).

c Inoue et al. (2017).

TABLE 3. Segregation of avirulence/virulence on wheat cultivars in random $F_{1}$ cultures derived from MZ5-1-6 $\times$ Br48

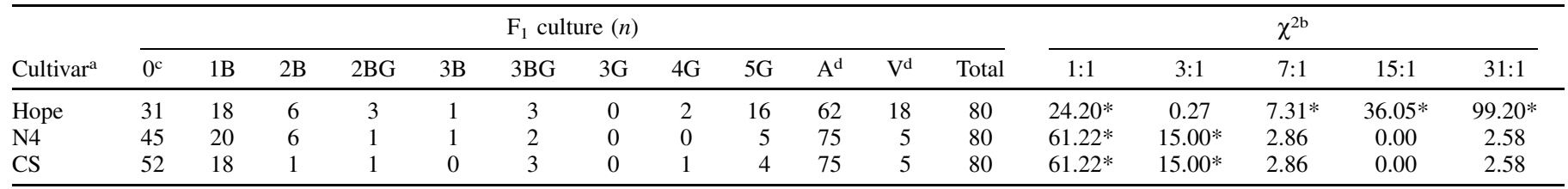

${ }^{a}$ N4 = Norin 4 and CS = Chinese Spring.

b An asterisk indicates a value significant at the $1 \%$ level.

c Infection type.

${ }^{\mathrm{d}} \mathrm{A}=$ avirulent (infection type $0-3 \mathrm{BG}$ ) and $\mathrm{V}=$ virulent (infection type 3G-5G). 
(Table 2). The thermal cycling conditions were composed of $98^{\circ} \mathrm{C}$ for $2 \mathrm{~min}$, followed by 45 cycles at $98^{\circ} \mathrm{C}$ for $10 \mathrm{~s}, 60^{\circ} \mathrm{C}$ for $10 \mathrm{~s}$, and $68^{\circ} \mathrm{C}$ for $15 \mathrm{~s}$.

Molecular mapping. Simple sequence repeat (SSR) markers for the P. oryzae genome (Zheng et al. 2008) were screened to find flanking markers of an avirulence gene. PCR for the SSR markers was performed using $2 \times$ Quick Taq HS DyeMix (TOYOBO) under the following conditions: 40 cycles of $10 \mathrm{~s}$ at $94^{\circ} \mathrm{C}, 30 \mathrm{~s}$ at the annealing temperature, and $30 \mathrm{~s}$ at $68^{\circ} \mathrm{C}$. Amplicons were electrophoresed in $6 \%$ polyacrylamide gel and stained with ethidium bromide. The MAPMAKER/EXP version 3.0 package was used for genetic mapping (Lander et al. 1987). The threshold for the logarithm-of-odds score was set at 3.0.

Genotyping of $\mathbf{F}_{\mathbf{1}}$ cultures. The $80 \mathrm{~F}_{1}$ cultures were subjected to genotyping at various loci. A portion of the PWT3 gene (242 bp) in each $\mathrm{F}_{1}$ was amplified with PWT3-HinfI-1F and PWT3-HinfI-1R (Table 2), and the resulting amplicon was digested with HinfI, which cleaved the B type (Br48 type) into two fragments (208 and $34 \mathrm{bp}$ ) but did not cleave the Ao' type (MZ5-1-6 type). Genotyping at the mating type locus was performed with A1/A5 (MAT1-1specific primers) and B16/B182 (MAT1-2-specific primers) (Table 2).

\section{RESULTS}

Segregation analysis of $\mathbf{F}_{\mathbf{1}}$ cultures. To identify avirulence genes of Eleusine isolates against common wheat, genetic analysis was performed using 80 random $\mathrm{F}_{1}$ cultures derived from a cross between an Eleusine isolate (MZ5-1-6) and a Triticum isolate $(\mathrm{Br} 48)$. The $\mathrm{F}_{1}$ cultures were sprayed onto three common wheat

A

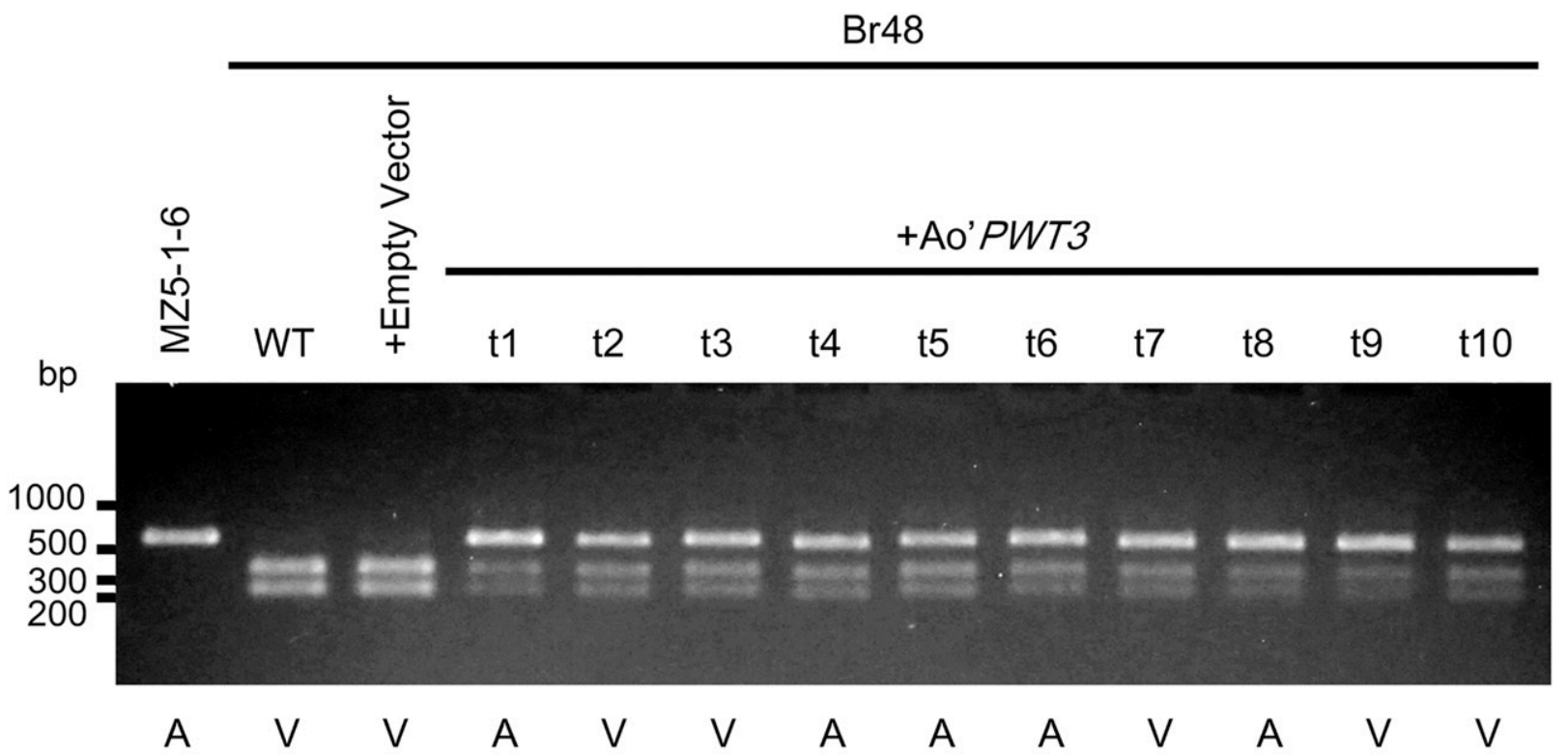

B

Br48

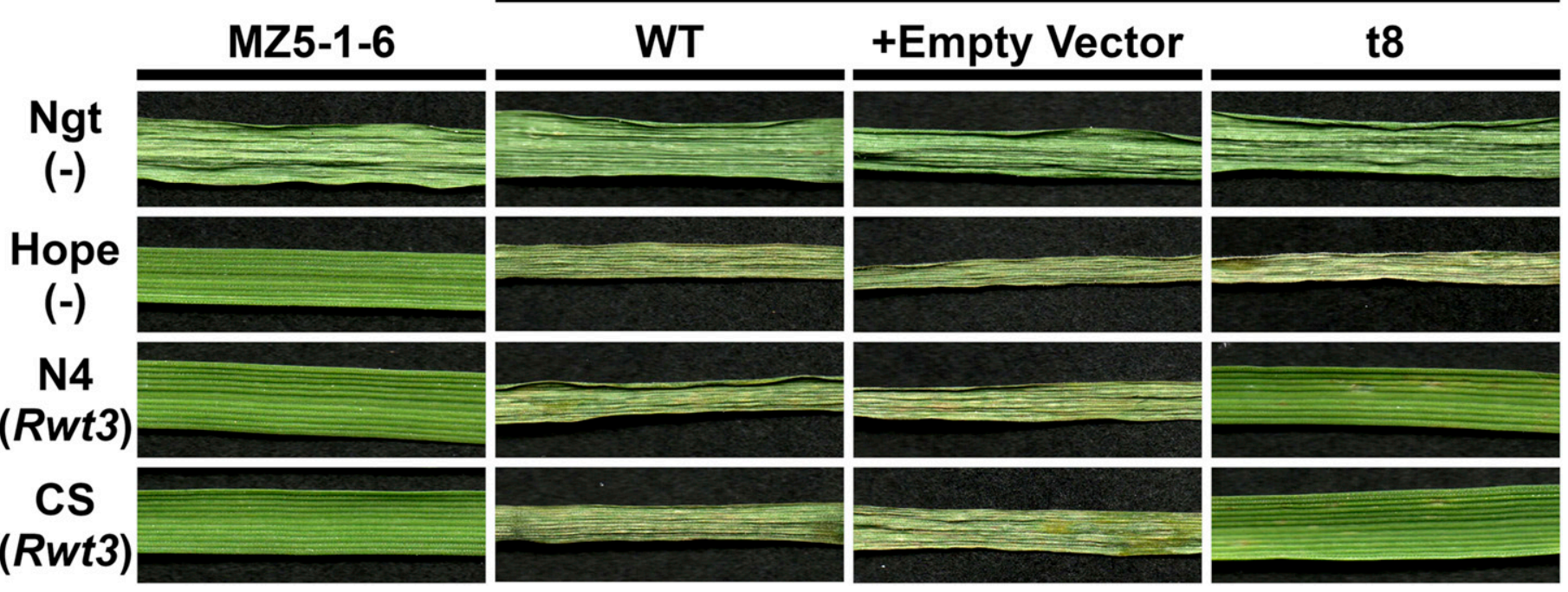

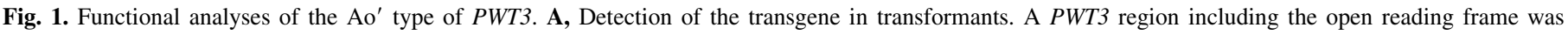

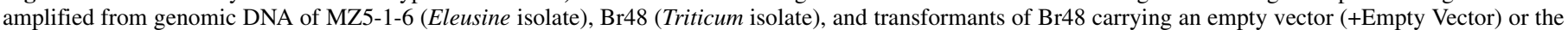

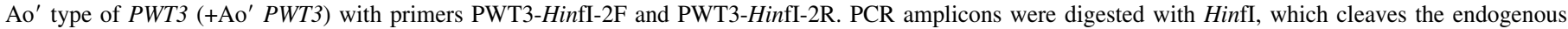

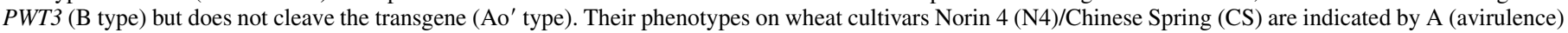

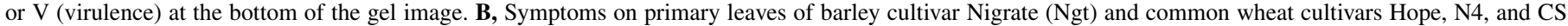
inoculated with MZ5-1-6, Br48, Br48+Empty Vector, and $t 8$ (Br48+Ao' PWT3), 4 days after inoculation. WT = wild type. 
cultivars: Hope, N4, and CS (Supplementary Table S1). On Hope, avirulent and virulent cultures segregated in a $3: 1$ ratio $(62: 18)$ (Table 3), suggesting that two genes are involved in the avirulence of MZ5-1-6 on this cultivar. On the other hand, the avirulence/ virulence segregations on $\mathrm{N} 4$ and $\mathrm{CS}$ (75:5) were not significantly deviated from the $7: 1,15: 1$, or $31: 1$ ratios (Table 3 ), suggesting that three or more avirulence genes are involved. The segregation patterns on N4 and CS were identical (Table 4), indicating that the same set of genes is involved in the avirulence on these two cultivars. All cultures avirulent on Hope were avirulent on N4/CS, and there were no cultures avirulent on Hope and virulent on N4/CS (Table 4), indicating that the two avirulence genes detected on Hope are also involved in the avirulence on N4/CS. These genes recognized by all tested cultivars were tentatively designated as $e A 1$ and $e A 2$. The $\mathrm{F}_{1}$ cultures virulent on Hope were composed of avirulent and virulent cultures on N4/CS (Table 4). The segregation (13:5) fitted a 3:1 ratio $\left(\chi^{2}=0.074\right)$, suggesting that at least two genes are involved in the avirulence on $\mathrm{CS} / \mathrm{N} 4$ in addition to $e A l$ and $e A 2$. These two genes detected only on N4/CS were tentatively designated as $e A 3$ and $e A 4$.

$\boldsymbol{e A 3}$ is the Ao' type of $\boldsymbol{P W T 3}$. Inoue et al. (2017) reported that $P W T 3$ homologs were widely distributed in the $P$. oryzae population. Avena and Lollium isolates carried a functional type of PWT3 designated as the Ao type, whereas Triticum isolates carried nonfunctional types of PWT3 such as the Atm type with an insertion of a transposable element (MGR583) into its promoter

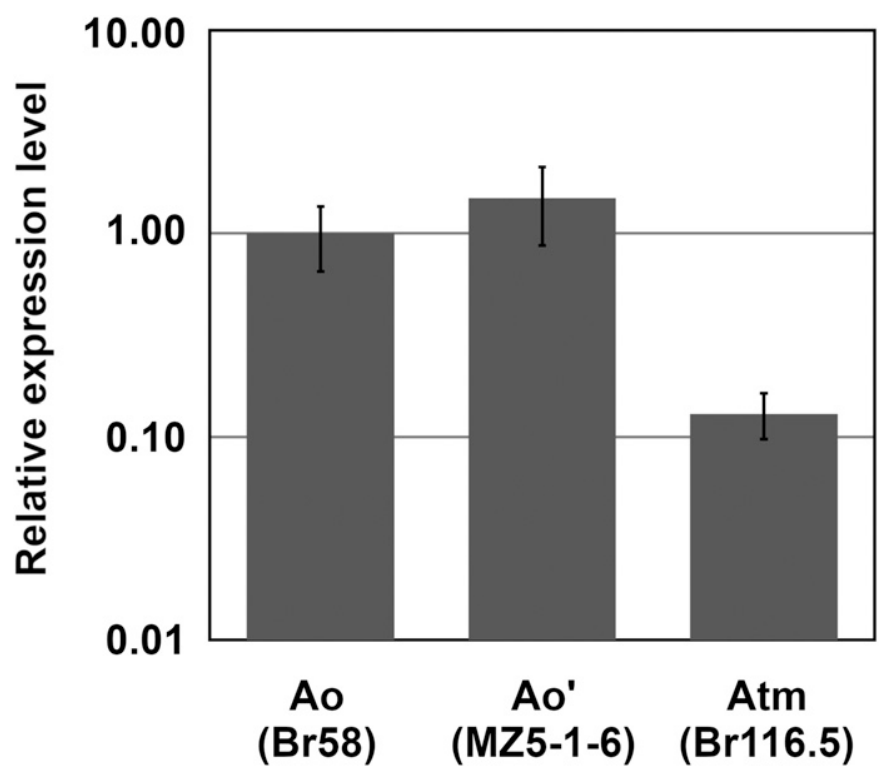

Fig. 2. In planta expression of the three types of $P W T 3$ at $30 \mathrm{~h}$ postinoculation. Expression levels of the Ao type in Br58 (functional), the Ao' type in MZ5-16 , and the Atm type in Br116.5 (nonfunctional) were measured by quantitative real-time PCR using the actin gene (MGG_03982) as an internal control. The vertical axis indicates the expression level relative to the Ao type. Bars indicate the standard deviation of three technical replicates. region, the Atc type with insertions of other transposable elements into its coding region, and the B type with nucleotide substitutions. Eleusine isolates (Z2-1 and MZ5-1-6) collected in Japan carried another type of PWT3 (Ao' type), which had the same coding sequence as in the Ao type but one single nucleotide polymorphism in its putative promoter region (Supplementary Fig. S1). To check whether the Ao' type was recognized by Rwt3 in N4 and CS, a complementation assay was performed. A fragment carrying the $\mathrm{Ao}^{\prime}$ type of PWT3 was amplified from MZ5-1-6, cloned into pBluescript, and then introduced into Br48 carrying the B type (nonfunctional). Among 10 transformants obtained, five gained avirulence on N4 and CS carrying Rwt3 without changing their virulence on Hope lacking Rwt3 (Fig. 1). The Ao' type was expressed at the same level as the functional Ao type, which was 10 times higher than that of the nonfunctional Atm type (Fig. 2). These results indicate that the $\mathrm{Ao}^{\prime}$ type functions as an avirulence gene recognized by $R w t 3$. This avirulence gene is recognized by $\mathrm{N} 4$ and CS but not by Hope (Fig. 1B), fulfilling the definition of $e A 3$ and $e A 4$. We assigned $e A 3$ to the Ao' type of PWT3.

We also examined the $P W T 3$ region in 34 EC-II isolates collected from various countries. Sequence analyses revealed that all EC-II isolates carried the Ao or Ao' types of PWT3 (Table 1). There were no EC-II isolates carrying nonfunctional $P W T 3$ or lacking $P W T 3$. It should be noted that the Ao' type was detected only in Japanese isolates but there was one exceptional Japanese isolate (G10-1) carrying the Ao type (Table 1).

Identification and mapping of $\boldsymbol{e A 4}$. To identify $e A 4$, we screened the $13 \mathrm{~F}_{1}$ cultures virulent on Hope and avirulent on N4/CS (Table 4) for noncarriers of $e A 3(=P W T 3)$. Of such $\mathrm{F}_{1}$ cultures, 200R72 carrying the MAT1-2 allele at the mating type locus was selected and backcrossed with $\mathrm{Br} 48$ (MAT1-1) to produce $\mathrm{BC}_{1} \mathrm{~F}_{1}$ cultures. When the resulting $\mathrm{BC}_{1} \mathrm{~F}_{1}$ cultures were sprayed onto test cultivars, avirulent and virulent cultures segregated in a 1:1 ratio (26:34) on N4/CS, whereas all cultures were virulent on Hope (Table 5). Here, we detected an avirulence gene that was recognized by N4/CS but not by Hope. This gene is apparently different from $e A 3$ because neither of their parents (200R72 and $\mathrm{Br} 48$ ) carries functional $P W T 3$. Therefore, we assigned $e A 4$ to this gene detected as a monofactorial segregation in the $\mathrm{BC}_{1} \mathrm{~F}_{1}$ population.

To clarify the chromosomal location of $e A 4$, molecular mapping with SSR markers (Zheng et al. 2008) was performed using the $\mathrm{BC}_{1} \mathrm{~F}_{1}$ population. The $e A 4$ gene was located on chromosome 2 and flanked by MGM51 and MGM195 with distances of 0.8 and $1.7 \mathrm{cM}$, respectively (Fig. 3).

Detection of the fifth gene, $\boldsymbol{e A 5}$. The segregation of $e A 4$ in the $F_{1}$ population was determined using its flanking markers, MGM51 and MGM195. $F_{1}$ cultures carrying the MZ5-1-6 alleles at both loci were considered to be $e A 4$ carriers, whereas those carrying the $\mathrm{Br} 48$ alleles at both loci were considered to be noncarriers of $e A 4$. All segregation data in the $\mathrm{F}_{1}$ population are summarized in Figure 4. $e A 4$ genotypes of five $\mathrm{F}_{1}$ cultures were not determined owing to recombination between the two molecular markers. It should be noted that two cultures, 200R30 (no. 74) and 200R79 (no. 75), were virulent on Hope but avirulent on N4/CS despite lacking functional alleles at the $P W T 3$ and $e A 4$ loci. This result indicates

TABLE 5. Segregation of avirulence/virulence on wheat cultivars in random $\mathrm{BC}_{1} \mathrm{~F}_{1}$ cultures derived from $200 \mathrm{R} 72 \times \mathrm{Br} 48$

\begin{tabular}{|c|c|c|c|c|c|c|c|c|c|c|c|c|c|c|}
\hline \multirow[b]{2}{*}{ Cultivar ${ }^{\mathrm{a}}$} & \multicolumn{11}{|c|}{$\mathrm{F}_{1}$ culture $(n)$} & \multicolumn{3}{|c|}{$\chi^{2 b}$} \\
\hline & $0^{c}$ & $1 \mathrm{~B}$ & $2 \mathrm{~B}$ & $2 \mathrm{BG}$ & $3 B$ & $3 B G$ & $3 \mathrm{G}$ & $4 \mathrm{G}$ & $5 \mathrm{G}$ & $\mathrm{A}^{\mathrm{d}}$ & $\mathrm{V}^{\mathrm{d}}$ & Total & $1: 1$ & $3: 1$ \\
\hline Hope & 0 & 0 & 0 & 0 & 0 & 0 & 6 & 11 & 43 & 0 & 60 & 60 & - & - \\
\hline N4 & 4 & 13 & 5 & 2 & 1 & 1 & 3 & 7 & 24 & 26 & 34 & 60 & 1.07 & $32.09 *$ \\
\hline $\mathrm{CS}$ & 9 & 14 & 2 & 0 & 1 & 0 & 7 & 8 & 19 & 26 & 34 & 60 & 1.07 & $32.09 *$ \\
\hline
\end{tabular}

a $\mathrm{N} 4$ = Norin 4 and CS = Chinese Spring.

b An asterisk indicates a value significant at the $1 \%$ level.

c Infection type.

d $\mathrm{A}=$ avirulent (infection type $0-3 \mathrm{BG}$ ) and $\mathrm{V}=$ virulent (infection type $3 \mathrm{G}-5 \mathrm{G}$ ). 
that these cultures should carry another avirulence gene that is recognized by N4/CS but not by Hope. This fifth gene was tentatively designated as $e A 5$.

\section{DISCUSSION}

This study revealed that at least five avirulence genes were involved in the incompatibility between an Eleusine isolate (belonging to EC-II) and standard wheat cultivars, CS and N4. This implies that "loss-of-function" mutations of at least five genes are needed for the Eleusine isolate to gain pathogenicity on wheat. Based on this result, we suggest that the possibility of host jumps of Eleusine isolates to wheat is very low or negligible compared with that of Lolium isolates to wheat. On Hope, however, only two avirulence genes were involved in the incompatibility. Hope is an exceptional cultivar that lacks resistance genes effective against Lolium and Avena isolates at the heading stage (Inoue et al. 2017). It may be advisable to avoid cultivation of such cultivars. It should be noted that this is a result with an EC-II isolate. Further studies are needed with the other lineage of the Eleusine pathotype, EC-I.

Inoue et al. (2017) demonstrated that the Ao type of $P W T 3$ played a key role in the incompatibility of Lolium and Avena isolates with wheat. The present study showed that one of the five avirulence genes of MZ5-1-6 was an allele (Ao' type) of PWT3. This allele was highly expressed in MZ5-1-6 (Fig. 2) and recognized by N4/CS
$\mathrm{cM}$

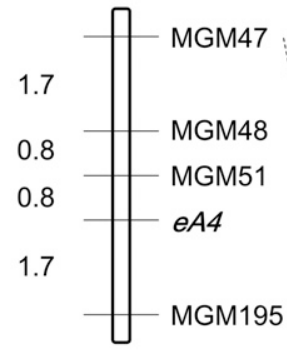

$200 \mathrm{R} 72 \times \mathrm{Br} 48$

$\mathrm{BC}_{1} \mathrm{~F}_{1} 60$ cultures
$\mathrm{CM}$

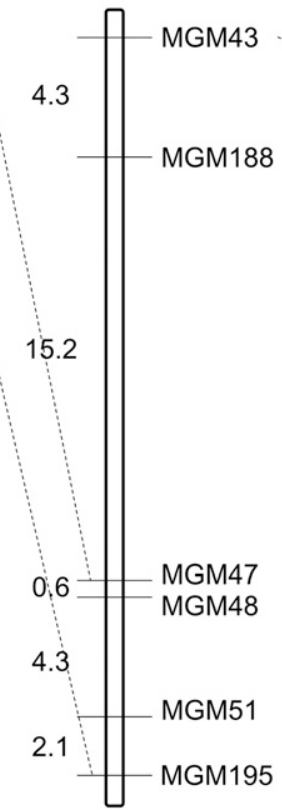

$\mathrm{MZ5}-1-6 \times \mathrm{Br} 48$ $F_{1} 80$ cultures
Chromosome 2

cM

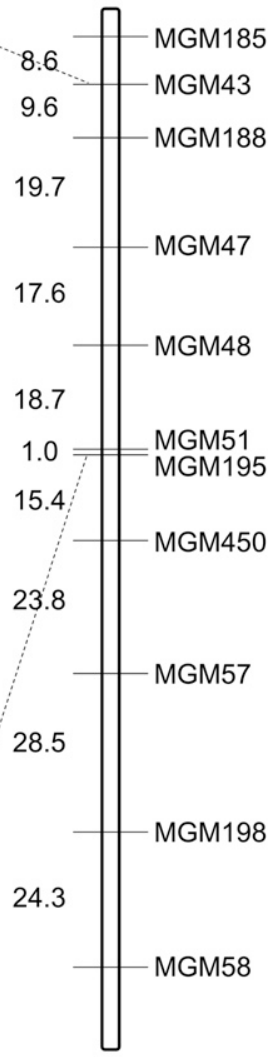

Guy11 x 2539

$F_{1} 228$ cultures

Zhen et al. (2008)
Fig. 3. Genetic map around $e A 4$ constructed using $80 \mathrm{~F}_{1}$ cultures derived from MZ5-1-6 $\times \mathrm{Br} 48$ and $60 \mathrm{BC}_{1} \mathrm{~F}_{1}$ cultures derived from $200 \mathrm{R} 72 \times \mathrm{Br} 48$. The original positions of simple sequence repeat markers reported by Zheng et al. (2008) are shown on the right. Genetic distances between markers are shown on the left of each linkage map in centimorgans. carrying its corresponding resistance gene, Rwt3 (Fig. 1). In addition, all EC-II isolates of the Eleusine pathotype carried functional alleles (Ao or Ao') of PWT3 (Table 1). These results suggest that $R w t 3$ is a very important gene that conditions resistance

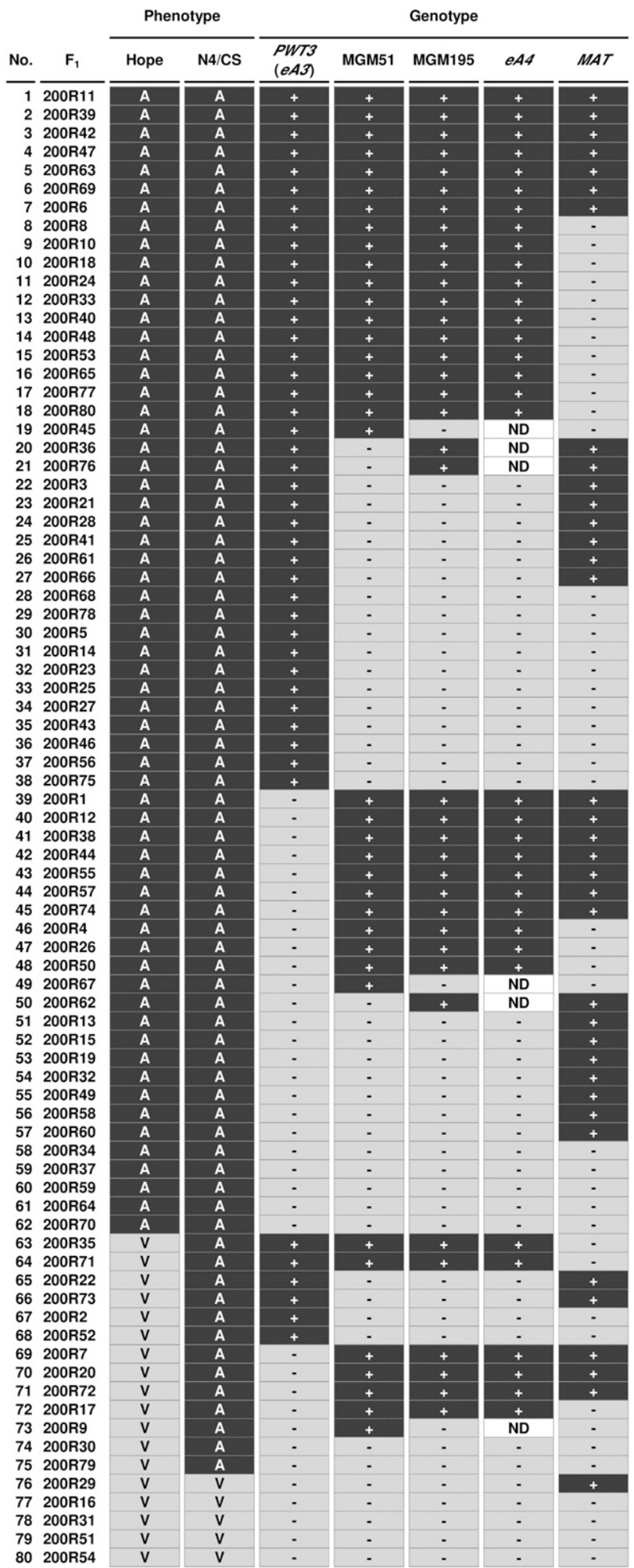

Fig. 4. Comparative analyses of segregation of phenotypes and molecular markers in the $80 \mathrm{~F}_{1}$ cultures derived from the cross between MZ5-1-6 and Br48. Phenotypes on wheat cultivars Hope, Norin 4 (N4), and Chinese Spring (CS) are indicated by A (avirulence) and V (virulence). MZ5-1-6 and Br48 types of each molecular marker are indicated by plus and minus signs, respectively. 
of wheat against a wide range of populations of $P$. oryzae-Eleusine, Lolium, and Avena isolates. This may be one reason why Rwt 3 has been maintained in the common wheat population in a high frequency (>75\%) (Inoue et al. 2017).

Finger millet is considered to have been domesticated in East Africa and transmitted to the Far East through India, Nepal, and China (Kato et al. 2000). Kato et al. (2000) suggested that Eleusine isolates may have also been transmitted from Africa to Japan with transmission of seeds of finger millet. The present study showed that, in the Eleusine isolates tested, all isolates from foreign countries were Ao carriers but that all isolates from Japan were Ao' carriers except one (Table 1). This result may be explained by assuming that the nucleotide substitution in the Ao' type (Supplementary Fig. S1) occurred soon after the EC-II isolate(s) entered Japan.

\section{ACKNOWLEDGMENTS}

We thank Miho Terada for production of the $F_{1}$ cultures by crossing the finger millet isolate with the wheat isolate.

\section{LITERATURE CITED}

Barr, M. E. 1977. Magnaporthe, Telimenella, and Hyponectria (Physosporellaceae). Mycologia 69:952-966.

Callaway, E. 2016. Devastating wheat fungus appears in Asia for first time. Nature 532:421-422.

Couch, B. C., and Kohn, L. M. 2002. A multilocus gene genealogy concordant with host preference indicates segregation of a new species, Magnaporthe oryzae, form M. grisea. Mycologia 94:683-693.

Cruz, C. D., and Valent, B. 2017. Wheat blast disease: Danger on the move. Trop. Plant Pathol. 42:210-222.

Gladieux, P., Condon, B., Ravel, S., Soanes, D., Maciel, J. L. N., Nhani, A., Jr., Chen, L., Terauchi, R., Lebrun, M.-H., Tharreau, D., Mitchell, T., Pedley, K. F., Valent, B., Talbot, N. J., Farman, M., and Fournier, E. 2018. Gene flow between divergent cereal- and grass-specific lineages of the rice blast fungus Magnaporthe oryzae. MBio 9:e01219-17.

Hyon, G.-S., Nga, N. T. T., Chuma, I., Inoue, Y., Asano, H., Murata, N., Kusaba, M., and Tosa, Y. 2012. Characterization of interactions between barley and various host-specific subgroups of Magnaporthe oryzae and M. grisea. J. Gen. Plant Pathol. 78:237-246.

Inoue, Y., Vy, T. T. P., Yoshida, K., Asano, H., Mitsuoka, C., Asuke, S., Anh, V. L., Cumagun, C. J. R., Chuma, I., Terauchi, R., Kato, K., Mitchell, T., Valent, B., Farman, M., and Tosa, Y. 2017. Evolution of the wheat blast fungus through functional losses in a host specificity determinant. Science 357:80-83.

Islam, M. T., Croll, D., Gladieux, P., Soanes, D. M., Persoons, A., Bhattacharjee, P., Hossain, M. S., Gupta, D. R., Rahman, M. M., Mahboob, M. G., Cook, N., Salam, M. U., Surovy, M. Z., Sancho, V. B., Maciel, J. L. N., Nhani, A., Jr., Castroagudín, V. L., de Assis Reges, J. T., Ceresini, P. C., Ravel, S., Kellner, R., Fournier, E., Tharreau, D., Lebrun, M.-H., McDonald, B. A., Stitt, T., Swan, D., Talbot, N. J., Saunders, D. G. O., Win, J., and Kamoun, S. 2016. Emergence of wheat blast in Bangladesh was caused by a South American lineage of Magnaporthe oryzae. BMC Biol. 14:84.

Kanamori, M., Kato, H., Yasuda, N., Koizumi, S., Peever, T. L., Kamakura, T., Teraoka, T., and Arie, T. 2007. Novel mating type-dependent transcripts at the mating type locus in Magnaporthe oryzae. Gene 403:6-17.
Kato, H., Yamaguchi, T., and Nishihara, N. 1976. The perfect state of Pyricularia oryzae Cav. in culture. Ann. Phytopathol. Soc. Jpn. 42:507-510.

Kato, H., Yamamoto, M., Yamaguchi-Ozaki, T., Kadouchi, H., Iwamoto, Y., Nakayashiki, H., Tosa, Y., Mayama, S., and Mori, N. 2000. Pathogenicity, mating ability and DNA restriction fragment length polymorphisms of Pyricularia populations isolated from Gramineae, Bambusideae and Zingiberaceae plants. J. Gen. Plant Pathol. 66:30-47.

Lander, E. S., Green, P., Abrahamson, J., Barlow, A., Daly, M. J., Lincoln, S. E., and Newburg, L. 1987. MAPMAKER: An interactive computer package for constructing primary genetic linkage maps of experimental and natural populations. Genomics 1:174-181.

Malaker, P. K., Barma, N. C. D., Tiwari, T. P., Collis, W. J., Duveiller, E., Singh, P. K., Joshi, A. K., Singh, R. P., Braun, H.-J., Peterson, G. L., Pedley, K. F., Farman, M. L., and Valent, B. 2016. First report of wheat blast caused by Magnaporthe oryzae pathotype triticum in Bangladesh. Plant Dis. 100: 2330 .

Murakami, J., Tosa, Y., Kataoka, T., Tomita, R., Kawasaki, J., Chuma, I., Sesumi, Y., Kusaba, M., Nakayashiki, H., and Mayama, S. 2000. Analysis of host species specificity of Magnaporthe grisea toward wheat using a genetic cross between isolates from wheat and foxtail millet. Phytopathology 90:1060-1067.

Tagle, A. G., Chuma, I., and Tosa, Y. 2015. Rmg7, a new gene for resistance to Triticum isolates of Pyricularia oryzae identified in tetraploid wheat. Phytopathology 105:495-499.

Takabayashi, N., Tosa, Y., Oh, H. S., and Mayama, S. 2002. A gene-for-gene relationship underlying the species-specific parasitism of Avena/Triticum isolates of Magnaporthe grisea on wheat cultivars. Phytopathology 92: 1182-1188.

Tanaka, M., Nakayashiki, H., and Tosa, Y. 2009. Population structure of Eleusine isolates of Pyricularia oryzae and its evolutionary implications. J. Gen. Plant Pathol. 75:173-180.

Tosa, Y., Hirata, K., Tamba, H., Nakagawa, S., Chuma, I., Isobe, C., Osue, J., Urashima, A. S., Don, L. D., Kusaba, M., Nakayashiki, H., Tanaka, A., Tani, T., Mori, N., and Mayama, S. 2004. Genetic constitution and pathogenicity of Lolium isolates of Magnaporthe oryzae in comparison with host species-specific pathotypes of the blast fungus. Phytopathology 94:454-462.

Tosa, Y., Osue, J., Eto, Y., Oh, H. S., Nakayashiki, H., Mayama, S., and Leong, S. A. 2005. Evolution of an avirulence gene, AVR1-CO39, concomitant with the evolution and differentiation of Magnaporthe oryzae. Mol. PlantMicrobe Interact. 18:1148-1160.

Ueyama, A., and Tsuda, M. 1975. Formation of the perfect state in culture of Pyricularia sp. from some graminaceous plants (preliminary report). Trans. Mycol. Soc. Jpn. 16:420-422.

Urashima, A. S., Igarashi, S., and Kato, H. 1993. Host range, mating type, and fertility of Pyricularia grisea from wheat in Brazil. Plant Dis. 77: 1211-1216.

Vy, T. T. P., Hyon, G.-S., Nga, N. T. T., Inoue, Y., Chuma, I., and Tosa, Y. 2014. Genetic analysis of host-pathogen incompatibility between Lolium isolates of Pyricularia oryzae and wheat. J. Gen. Plant Pathol. 80:59-65.

Yaegashi, H., and Nishihara, N. 1976. Production of the perfect stage in Pyricularia from cereals and grasses. Ann. Phytopathol. Soc. Jpn. 42: 511-515.

Zhang, N., Luo, J., Rossman, A. Y., Aoki, T., Chuma, I., Crous, P. W., Dean, R., de Vries, R. P., Donofrio, N., Hyde, K. D., Lebrun, M.-H., Talbot, N. J., Tharreau, D., Tosa, Y., Valent, B., Wang, Z., and Xu, J.-R. 2016. Generic names in Magnaporthales. IMA Fungus 7:155-159.

Zheng, Y., Zhang, G., Lin, F., Wang, Z., Jin, G., Yang, L., Wang, Y., Chen, X., Xu, Z., Zhao, X., Wang, H., Lu, J., Lu, G., and Wu, W. 2008. Development of microsatellite markers and construction of genetic map in rice blast pathogen Magnaporthe grisea. Fungal Genet. Biol. 45:1340-1347. 